\title{
ESTUDO PALINOTAXONÔMICO DE ESPÉCIES DE SCHEFFLERA (Araliaceae) da Região Sudeste do Brasil
}

\author{
Pedro Fiaschi ${ }^{1,3}$, Maria Amelia Vitorino da Cruz-Barros ${ }^{2}$ \\ \& Angela Maria da Silva Correa ${ }^{2}$
}

\begin{abstract}
Resumo
(Estudo palinotaxonômico de espécies de Schefflera (Araliaceae) da Região Sudeste do Brasil) Foi estudada a morfologia dos grãos de pólen de 18 espécies de Schefflera ocorrentes na Região Sudeste do Brasil: $S$. angustissima, S. calva, S. capixaba, S. cordata, S. fruticosa, S. gardneri, S. glaziovii, S. longipetiolata, S. lucumoides, S. macrocarpa, S. malmei, S. morototoni, S. selloi, S. spruceana, S. succinea, S. villosissima, $S$. vinosa e Schefflera aff. varisiana. Os grãos de pólen estudados são geralmente médios, raramente pequenos, com âmbito triangular a subtriangular, anguloaperturados, oblato-esferoidais a prolato-esferoidais, 3colporados, exina reticulada heterobrocada ou rugulado-reticulada. Relações filogenéticas entre algumas das espécies estudadas são discutidas com base nos resultados obtidos.
\end{abstract}

Palavras-chave: Araliaceae, Didymopanax, grãos de pólen, Schefflera.

\section{Abstract}

(Palynotaxonomic study of southeastern Brazilian species of Schefflera (Araliaceae)) Morphological studies of the pollen grains of 18 species of Schefflera from Southeastern Brazil were carried out: S. angustissima, S. calva, S. capixaba, S. cordata, S. fruticosa, S. gardneri, S. glaziovii, S. longipetiolata, S. lucumoides, S. macrocarpa, S. malmei, S. morototoni, S. selloi, S. spruceana, S. succinea, S. villosissima, S. vinosa e Schefflera aff. varisiana. The pollen grains analysed are medium, rarely small, with triangular to subtriangular amb, anguloaperturate, oblate spheroidal to prolate spheroidal, 3-colporate, and reticulate heterobrochate or rugulate-reticulate ornamentation. Phylogenetic relationships among some of the studied species are discussed based on the obtained results.

Key words: Araliaceae, Didymopanax, pollen grains, Schefflera.

\section{INTRODUÇÃOO}

Schefflera J.R.Forst. \& G.Forst. é o maior gênero de Araliaceae, com 650-900 espécies distribuídas principalmente em regiões tropicais, especialmente em formações montanhosas como os Andes, montanhas da Malásia e Indonésia, Madagascar, ilhas da Melanésia e Planalto das Guianas (Frodin 1995a, 2004; Frodin \& Govaerts 2003; Plunkett et al. 2005).

A circunscrição atual de Schefflera é inconsistente a partir de análises filogenéticas, uma vez que constitui um grupo polifilético composto por 900 espécies agrupadas em cinco linhagens evolutivas independentes, referidas como os clados Asiático (200-300 spp.), Neotropical (ca. 300 spp.), Africano-Madagascar (ca. 50 spp.), Pacífico (ca. 45 spp.) e Schefflera sensu stricto (8 spp., incluindo $S$. digitata, a espécie tipo do gênero) (Lowry et al. 2004, Plunkett et al. 2004,
2005). Das cerca de 300 espécies neotropicais de Schefflera, estima-se que 60 ocorram no Brasil, distribuídas em sua maioria no Planalto Central (principalmente ao longo da Cadeia do Espinhaço em Minas Gerais), Planalto das Guianas, e Mata Atlântica do Sudeste (Frodin 1993, 1995a, 1997; Fiaschi 2004; Fiaschi \& Pirani 2005a, b, 2007).

Há poucos estudos taxonômicos recentes sobre as espécies brasileiras do gênero, dentre os quais destacam-se algumas monografias direcionadas a levantamentos florísticos de áreas restritas (Frodin 1995b, 1997; Jung 1981; Jung-Mendaçolli \& Cabral 2000; Fiaschi \& Pirani 2005c, 2007) ou descrições de espécies novas encontradas no país (Maguire et al. 1984; Frodin 1993; Fiaschi 2004; Fiaschi \& Pirani 2005a, b; Fiaschi et al. 2008).

Apesar da enorme variabilidade macromorfológica dos grãos de pólen dos representantes

Artigo recebido em 06/2008. Aceito para publicação em 11/2008.

${ }^{1}$ Department of Biology, Virginia Commonwealth University, Richmond, VA 23284-2012, U.S.A.

${ }^{2}$ Instituto de Botânica, C.P. 3005, 01061-970, São Paulo, SP, Brasil.

${ }^{3}$ Autor para correspondência: pedrofiaschi@ hotmail.com 
de Schefflera como um todo (e.g., Tseng \& Shoup 1978), ainda há poucos estudos abordando os aspectos taxonômicos da morfologia polínica das espécies brasileiras. Algumas exceções são os trabalhos de Salgado-Labouriau (1973), Shoup \& Tseng (1977) e Melhem \& Bissa (1985).

Este estudo visou analisar a morfologia polínica de espécies de Schefflera ocorrentes na Região Sudeste do Brasil, constituindo uma das primeiras contribuições para o reconhecimento dos tipos polínicos encontrados em espécies neotropicais do gênero.

\section{Material e Métodos}

Os grãos de pólen utilizados para a análise foram retirados de botões florais de flores perfeitas em pré-antese. Uma vez que duas das espécies estudadas ( $S$. aff. varisiana e S. spruceana) possuem apenas flores perfeitas, decidiu-se incluir apenas grãos de pólen provenientes dessas flores no presente estudo.

As exsicatas das quais foram retirados os botões florais estão depositadas nos herbários BHCB, ESA, MBM, RB, SP, SPF, UB e UEC (acrônimos de acordo com Holmgren et al. 1990).

Para cada espécie foram estudados, sempre que possível, os grãos de pólen de cinco espécimes (seis em Schefflera vinosa). Um destes foi escolhido como material padrão (assinalado por um asterisco), no qual foram efetuadas todas as medidas e observações para a caracterização dos grãos de pólen. Os demais foram utilizados como material de comparação, para a verificação da variabilidade polínica de cada uma das espécies incluídas.

\section{Material examinado}

Schefflera angustissima (Marchal) Frodin. BRASIL. SÃOPAULO: Bananal, 1100m alt., 14.IV.2000, A. Costa et al. 742 (SPF); Bertioga, s.d., S. E. Martins 601 (SPF); idem, 10.XII.2000, P. Fiaschi \& A. Q. Lobão

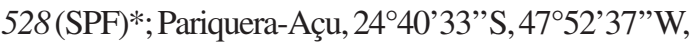
12.XI.1995, N. M. Ivanauskas 559 (ESA); São Paulo, Jardim Botânico, 23.IX.1931, O. Handro 96 (SP).

S. calva (Cham.) Frodin \& Fiaschi. BRASIL. MINAS GERAIS: Santana do Riacho, 11.I.1998, J. R. Pirani et al. CFSC 11027 (SPF); São Roque de Minas,
15.XII.1998, M. A. Farinaccio \& E. M. Campos Filho 228 (SPF)*.

S. capixaba Fiaschi. BRASIL. ESPÍRITO SANTO: Cariacica, $20^{\circ} 17^{\prime} 28^{\prime \prime} \mathrm{S}, 40^{\circ} 31^{\prime} 20^{\prime \prime}-40^{\circ} 31^{\prime} 55^{\prime}$ 'W, 680750m alt., 8.III.2001, P. Fiaschi et al. 690 (SPF, holótipo)*.

S. cordata (Taub.) Frodin \& Fiaschi. BRASIL. MINAS GERAIS: Diamantina, $18^{\circ} 07^{\prime} \mathrm{S}, 43^{\circ} 32^{\prime} \mathrm{W}$, 18.VI.2000, P. Fiaschi \& F. N. Costa 314 (SPF)*.

S. fruticosa Fiaschi \& Pirani. BRASIL. MINAS GERAIS: Jaboticatubas, 15.VI.2000, P. Fiaschi \& F. N. Costa 286 (SPF, holótipo)*; idem, 12.II.1996, N. Roque et al. 104 (SPF).

S. gardneri (Seem.) Frodin \& Fiaschi. BRASIL. MINAS GERAIS: Gouveia, 18³6'S, 4354'W, IV.1982, A. Furlan et al. CFCR 3224 (SPF); Itacambira, 1704'57'S, 4318'45"W, 1300m alt., 17.V.1998, J.R. Pirani etal. 4383 (SPF); Joaquim Felício, 31.VIII.1985, T. B. Cavalcanti et al. CFCR 8073 (SPF)*.

S. glaziovii (Taub.) Frodin \& Fiaschi. BRASIL. MINAS GERAIS: Santana de Pirapama, $18^{\circ} 55^{\prime} \mathrm{S}, 43^{\circ} 54^{\prime} \mathrm{W}$, 20.VI.2000, P. Fiaschi \& F. N. Costa 330 (SPF); Santana do Riacho, $19^{\circ} 04^{\prime} \mathrm{S}, 43^{\circ} 42^{\prime} \mathrm{W}, 1090 \mathrm{~m}$ alt., 5.III.1998, J. R. Pirani et al. 4247 (SPF)*; idem, 21.VI.2000, P. Fiaschi \& F. N. Costa 343 (SPF).

S. longipetiolata (Pohl ex DC.) Frodin \& Fiaschi. BRASIL. MINAS GERAIS: Descoberto, 23.II.2001, P. Fiaschi \& Castro 617 (SPF)*; Matão, 23. IX.1984, P. M. Andrade \& M. A. Lopes 348 (BHCB); idem, 20.XI.1984, P. M. Andrade \& M. A. Lopes 511 (BHCB). Rio de Janeiro: Santa Maria Madalena, $21^{\circ} 58^{\prime}$ 'S, 41 ${ }^{\circ} 58^{\prime}$ 'W, 700-800m alt., 26.VI.1987, C. Farney et al. 1444 (RB); idem, California-Valerio (Cachoeiras), 20.VI.1922, J. G. Kuhlmann s.n. (RB 21344).

S. lucumoides (Decne. \& Planch. ex Marchal) Frodin $\&$ Fiaschi. BRASIL. MINAS GERAIS: Itabira do Campo, 8.III.1994, W.A. Teixeira s.n. (BHCB 26131)*; idem, $20^{\circ} 14^{\prime}$ 'S, 434ㅇ' 'W, $1300 \mathrm{~m}$ alt., 14.XI.1987, $R$. F. Pinto s.n. (BHCB 11726); idem, 24.X.1994, W. A. Teixeira s.n. (BHCB 26039).

S. macrocarpa (Cham. \& Schltdl.) Frodin. BRASIL. BAHIA: Caetité, $14^{\circ} 08^{\prime} 48^{\prime \prime} S, 42^{\circ} 32^{\prime} 29^{\prime \prime}$ W, 960 m alt., 11.II.1997, M. L. Guedes et al. PCD 5421 (SPF). DISTRITOFEDERAL: 7.IV.2000, C. Proença etal. 2162 (SPF). GOIÁS: Itameri, $17^{\circ} 03^{\prime} 15^{\prime \prime} \mathrm{S}, 47^{\circ} 45^{\prime} 02^{\prime \prime} \mathrm{W}$, $900 \mathrm{~m}$ alt., 31.I.2000, P. Fiaschi \& A. C. Marcato 140 (SPF)*. MINAS GERAIS: Santana do Riacho, 18.II.1982, A. M. Giulietti et al. CFSC 7909 (SPF).

S. malmei (Harms) Frodin. BRASIL. MATOGROSSO: Tangará da Serra, $14^{\circ} 23^{\prime}$ 'S, 58 $18^{\circ}$ 'W, 1.IX.1986, $M$. M. Santos 203 (MBM)*. 
S. morototoni (Aubl.) Maguire, Steyermark \& Frodin. BRASIL. SÃOPAULO: I.1884, J. Saldanha 8517(R)*.

S. selloi (Marchal) Frodin \& Fiaschi. BRASIL. BAHIA: Belmonte, 12.I.1985, L. A. Mattos-Silva et al. 1807 (UEC); Maraú, 17.I.1967, R. P. Belém \& R.S. Pinheiro 3154 (UEC). ESPÍRITO SANTO: Guarapari, 20³2'53's, 40²3'34'W, 20-25m alt., 5.III.2001, P. Fiaschi et al. 640 (SPF)*; Vila Velha, 14.I.1975, A. L. Peixoto et al. 379 (RB).

S. spruceana (Seem.) Maguire, Steyermark \& Frodin. BRASIL. AMAZONAS: São Felipe, 23.IX.1952, $R$. L. Fróes 28702 (SP)*.

S. succinea Frodin \& Fiaschi. BRASIL. RIO DE JANEIRO: Nova Friburgo, $22^{\circ} 00^{\prime} \mathrm{S}, 42^{\circ} 03^{\prime} \mathrm{W}, 1100$ m alt., 26.XI.1986, G. Martinelli 11929 (SP)*; Santa Maria Madalena, $1800 \mathrm{~m}$ alt., 30.VI.1989, G. Martinelli et al. 13395 (RB).

S. villosissima Fiaschi \& Pirani. BRASIL. MINAS GERAIS: ca. $19 \mathrm{~km} \mathrm{~N}$ of Serro, on road (MG 2) to Diamantina, 1200m alt., 24.II.1968, H. S. Irwin et al. 20809 (UB)*.

S. vinosa (Cham. \& Schltdl.) Frodin \& Fiaschi. BRASIL. BAHIA: Lençóis, $12^{\circ} 33$ '38”'S, 41 23'29”'W, 1000m alt., 10.III.1996, A.A. Conceição et al. PCD 2218 (SPF); idem, Rio de Contas, 10.IV.1999, R.C. Forzza et al. 1148 (SPF). MINAS GERAIS: Diamantina, 3.VIII.1985, J. R. Pirani et al. CFCR 7932 (SPF); Rio Vermelho, 14.VII.1984, R. M. Harley s.n. (SPF 33280). SÃOPAULO: Cristália, 8.VII.2000, P. Fiaschi \& A. V. Christianini 350 (SPF); Itararé, 30.VII.1999, P. Fiaschi 30 (SPF)*.

$S$. aff. varisiana Frodin. BRASIL. BAHIA: Abaíra, 13'16'S, 41 ${ }^{\circ} 53^{\prime} \mathrm{W}, 1500-1650 \mathrm{~m}$ alt., 29.XII.1991, E. Nic-Lughadha et al. H 50219 (SPF)*; idem, $13^{\circ} 15^{\prime} \mathrm{S}, 41^{\circ} 55^{\prime} \mathrm{W}, 1800-1850 \mathrm{~m}$ alt., 22.III.1992, $T$. Laessoe \& T. Silva H 53300 (SPF); idem, 13ำ' 's; $41^{\circ} 55^{\prime} \mathrm{W}, 1650 \mathrm{~m}$ alt., 3.II.1992, B. Stannard et al. $H$ 51151 (SPF); idem, $1670 \mathrm{~m}$ alt., 14.II.1992, R. M. Harley et al. H 52026 (SPF); idem, 1316'S, 41 ${ }^{\circ} 54^{\prime} \mathrm{W}, 1700-1800 \mathrm{~m}$ alt., 24.II.1992, P. T. Sano \& T. Laessoe H 52190 (SPF).

Das espécies estudadas, apenas Schefflera spruceana não ocorre na Região Sudeste do Brasil. A inclusão dessa espécie deu-se com o intuito de verificar a possível semelhança de seus grãos de pólen com os de Schefflera aff. varisiana, uma vez que ambas fazem parte do Grupo "Crepinella” (Frodin 1995a).

Para a análise em microscopia óptica (MO), os grãos de pólen foram preparados pelo método de acetólise (Erdtman 1960) e fotografados com o auxílio de um microscópio óptico Olympus BX-50 acoplado a uma câmera de vídeo Olympus e um microcomputador PC, utilizando-se o software Image Pro-Plus 3. Para a análise de microscopia eletrônica de varredura (MEV), foram seguidas as etapas citadas em Melhem et al. (2003) para grãos de pólen acetolisados. As eletromicrografias foram obtidas em um microscópio Zeiss DSM 970, no Laboratório de Microscopia Eletrônica do Instituto de Biociências da Universidade de São Paulo. A terminologia utilizada para a descrição dos grãos de pólen seguiu Barth \& Melhem (1988) e Punt et al. (2007).

As medidas dos diâmetros dos materiais padrão foram feitas em 25 grãos de pólen tomados ao acaso, e distribuídos em pelo menos três lâminas visando uma uniformidade da amostra (Salgado-Labouriau et al. 1965). Foram feitas, sempre que possível, 10 medidas da abertura, lado do apocolpo, ornamentação e espessura das camadas da exina, o mesmo ocorrendo para as medidas dos diâmetros dos grãos de pólen dos materiais de comparação. Para a contagem e medida das perfurações, dos lumens e seus respectivos muros, foi estabelecida uma área de ca. $50 \mu^{2}$, na vista polar dos grãos de pólen. Foram obtidos valores dos seguintes parâmetros estatísticos: média aritmética $(\bar{x})$ desvio padrão da média $\left(s_{\bar{x}}\right)$, desvio padrão da amostra $(s)$, e o coeficiente de variabilidade (V). As comparações de duas médias foram feitas através da análise do intervalo de confiança (IC) a 95\% (Vieira 1981).

\section{Resultados e Discussão}

Os grãos de pólen das espécies estudadas de Schefflera (Figs. 1-3) são morfologicamente similares, podendo ser descritos como pequenos (S. malmei, S. morototoni e S. villosissima) a médios (Tabs. 1-2); âmbito triangular; anguloaperturados; área polar pequena a muito pequena (S. spruceana)(Fig. 3i); oblatoesferoidais a prolato-esferoidais (S. capixaba, S. spruceana e $S$. aff. varisiana); 3-colporados, colpos longos a muito longos, estreitos, pouco 
nítidos e de difícil visualização e mensuração (Tab. 3), com margem delgada; extremidades dos colpos arredondadas ou afiladas; endoabertura alongada, de difícil visualização e mensuração devido à ornamentação da exina (Tab. 3), com constrição mediana em $S$. capixaba e $S$. fruticosa; exina reticulada heterobrocada ou rugulado-reticulada ( $S$. aff. varisiana e $S$. spruceana), nexina mais espessa que a sexina (exceção em $S$. angustissima e S. capixaba) (Tab. 4). Sob $\mathrm{MEV}$, em S. succinea, a ornamentação da sexina no mesocolpo é caracterizada pela presença de retículos com muros curvos e entrelaçados (Fig. 31), enquanto que no pólo a ornamentação, quando vista sob MO (Fig. 3mn), parece seguir o padrão reticulado heterobrocado. Nas espécies reticuladas heterobrocadas a sexina apresenta-se composta por retículos, microrretículos e perfurações em quantidades variáveis (Tab. 5).
As análises realizadas nos permitiram separar as espécies estudadas em dois padrões polínicos, no que diz respeito à ornamentação da sexina.

O primeiro padrão (Tipo 1), caracterizado por apresentar grãos de pólen com exina reticulada (e.g., Figs. 1c-e, 3c-f), é encontrado na maioria das espécies estudadas com distribuição geográfica predominante no Sudeste do Brasil, tais como S. angustissima, S. calva, S. longipetiolata, S. macrocarpa, S. vinosa, entre outras (Schefflera grupo "Didymopanax", sensu Frodin 1995a). Esse tipo apresenta grãos de pólen oblatoesferoidais, exceto o material padrão de $S$. capixaba e o espécime Furlan et al. CFCR 3224 de $S$. gardneri, que apresentou grãos de pólen prolato-esferoidais; já os espécimens Giulietti et al. CFSC 7009, de S. macrocarpa, e Belém \& Pinheiro 3154, de S. selloi, apresentaram grãos de pólen suboblatos.

Tabela 1 - Caracterização morfológica dos grãos de pólen de espécies de Schefflera $(\mathrm{P}=$ diâmetro polar em vista equatorial; $\mathrm{E}=$ diâmetro equatorial $\mathrm{em}$ vista equatorial).

\begin{tabular}{lllll}
\hline Espécie & Tamanho & P/E & Exina & Figuras \\
\hline S. angustissima & Médio & 0,95 & Reticulada heterobrocada & $1 \mathrm{a}-\mathrm{f}$ \\
S. calva & Médio & 0,89 & Reticulada heterobrocada & $1 \mathrm{~g}-\mathrm{l}$ \\
S. capixaba & Médio & 1,01 & Reticulada heterobrocada & $1 \mathrm{~m}-\mathrm{p}$ \\
S. cordata & Médio & 0,92 & Reticulada heterobrocada & $1 \mathrm{q}-\mathrm{t}$ \\
S. fruticosa & Médio & 0,96 & Reticulada heterobrocada & $1 \mathrm{u}-\mathrm{z}$ \\
S. gardneri & Médio & 0,95 & Reticulada heterobrocada & $2 \mathrm{a}-\mathrm{d}$ \\
S. glaziovii & Médio & 0,93 & Reticulada heterobrocada & $2 \mathrm{e}-\mathrm{h}$ \\
S. longipetiolata & Médio & 0,90 & Reticulada heterobrocada & $2 \mathrm{i}-1$ \\
S. lucumoides & Médio & 0,95 & Reticulada heterobrocada & $2 \mathrm{~m}-\mathrm{p}$ \\
S. macrocarpa & Médio & 0,91 & Reticulada heterobrocada & $2 \mathrm{q}-\mathrm{v}$ \\
S. malmei & Pequeno & 0,93 & Reticulada heterobrocada & $2 \mathrm{w}-\mathrm{z}$ \\
S. morototoni & Pequeno & 0,95 & Reticulada heterobrocada & $2 \mathrm{a}-\mathrm{d}$ \\
S. selloi & Médio & 0,89 & Reticulada heterobrocada & $3 \mathrm{a}-\mathrm{f}$ \\
S. spruceana & Médio & 1,01 & Rugulado-reticulada & $3 \mathrm{~g}-\mathrm{i}$ \\
S. succinea & Médio & 0,95 & Reticulada heterobrocada & $3 \mathrm{j}-\mathrm{n}$ \\
S. villosissima & Pequeno & 0,95 & Reticulada heterobrocada & $3 \mathrm{o}-\mathrm{r}$ \\
S. vinosa & Médio & 0,88 & Reticulada heterobrocada & $3 \mathrm{~s}-\mathrm{v}$ \\
S. aff. varisiana & Médio & 1,13 & Rugulado-reticulada & $3 \mathrm{w}-\mathrm{y}$ \\
\hline
\end{tabular}



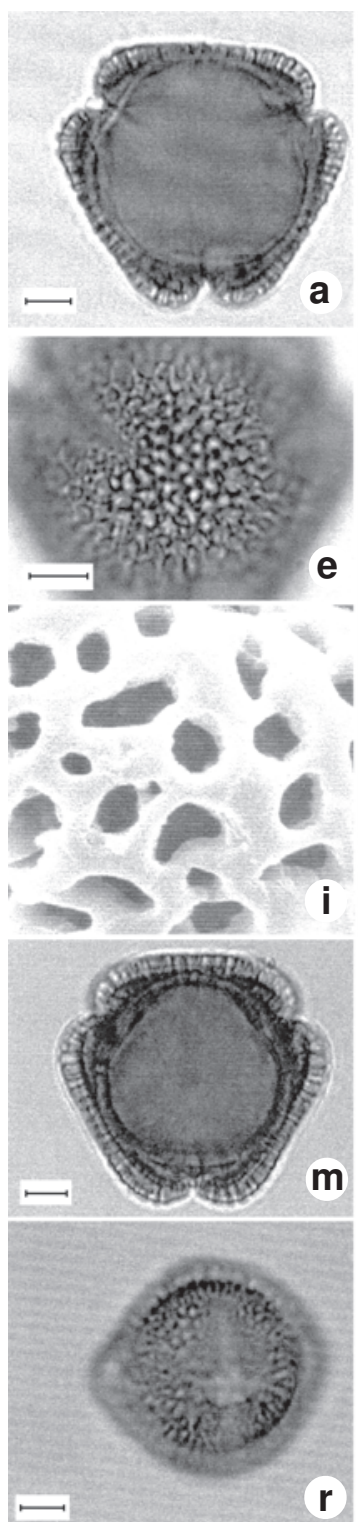

r

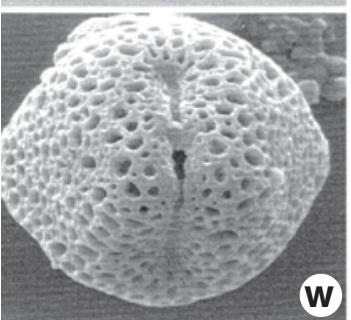

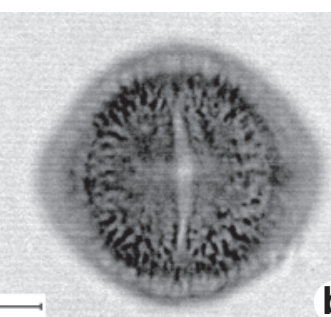
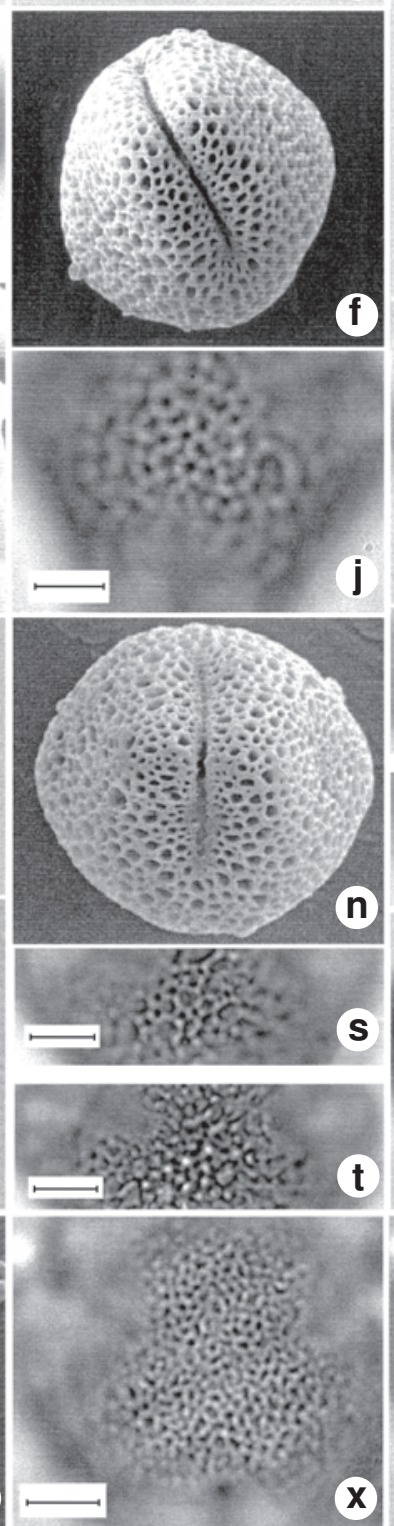

b
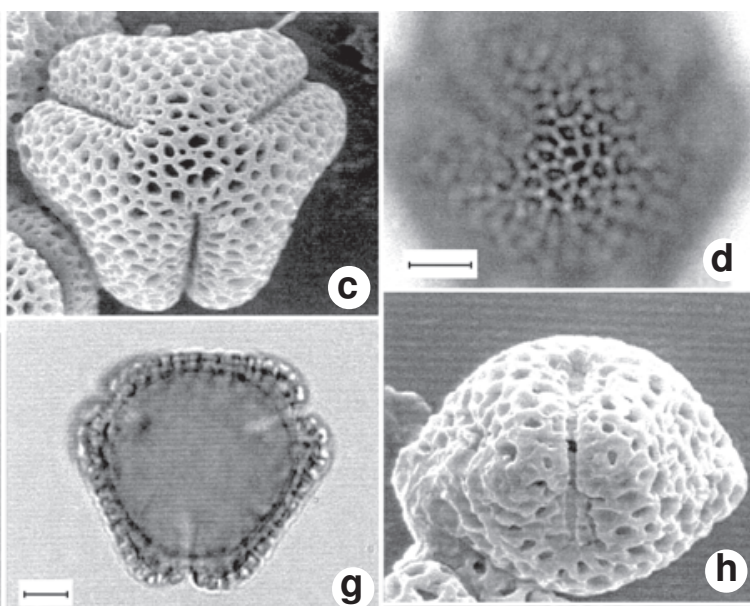

g
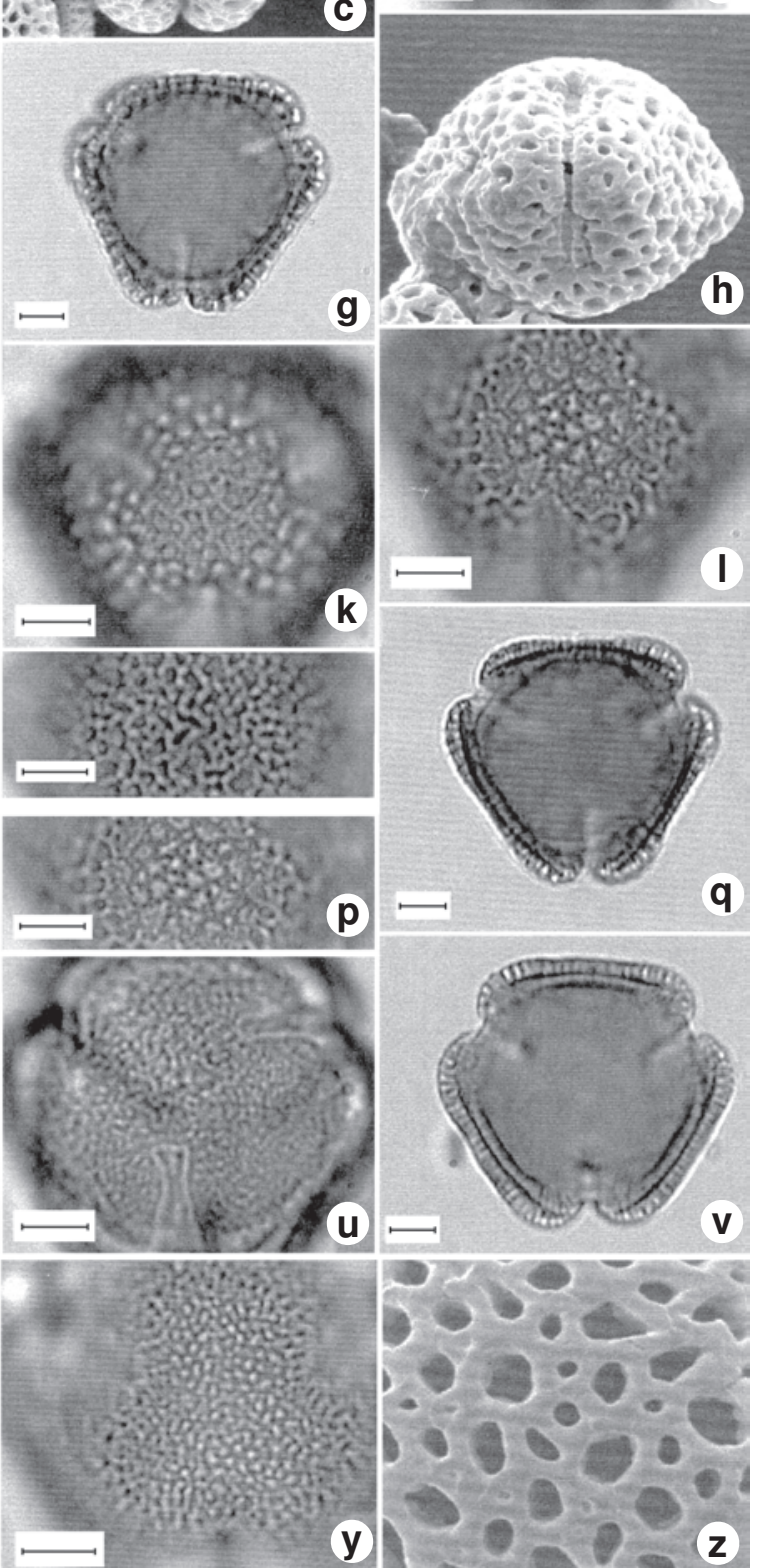

Figura 1 -Fotomicrografias e eletromicrografias dos grãos de pólen de Schefflera. a-f. S. angustissima - a. corte óptico em vista polar (VP); b. abertura em vista equatorial (VE); c. vista polar em MEV (aumento 3.000x); d-e. análise L.O. em dois níveis de focalização; f. vista equatorial em MEV (aumento 3.000x). g-1. S. calva - g. corte óptico em VP; h. vista equatorial em MEV (aumento 3.000x); i. detalhe da ornamentação no apocolpo em MEV (aumento 10.000x); j-1. análise L.O. em três níveis de focalização. m-p. S. capixaba - m. corte óptico em VP; n. vista equatorial em MEV (aumento 3.000x); o-p. análise L.O. em dois níveis de focalização. q-t. S. cordata - q. corte óptico em VP; r. abertura em VE; s-t. análise L.O. em dois níveis de focalização. u-z. S. fruticosa - u. detalhe do apocolpo em VP; v. corte óptico em VP; w. vista equatorial em MEV (aumento 3.000x); x-y. análise L.O. em dois níveis de focalização; z. detalhe da ornamentação no apocolpo (aumento 10.000x). Escala =5 $\mu \mathrm{m}$. 


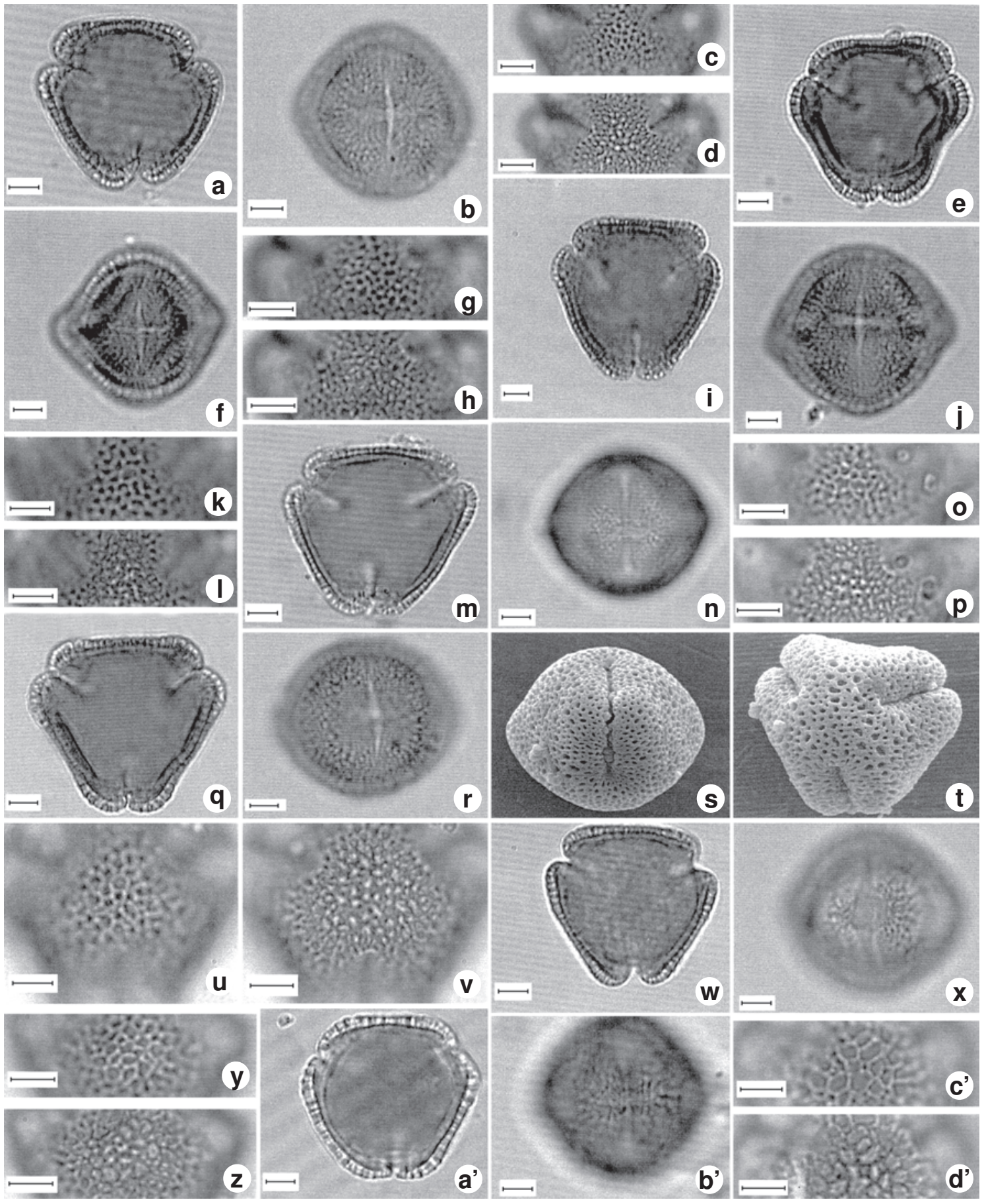

Figura 2 - Fotomicrografias e eletromicrografias dos grãos de pólen de Schefflera. a-d. S. gardneri-a. corte óptico em VP; b. abertura em VE; c-d. análise L.O. em dois níveis de focalização. e-h. S. glaziovii - e. corte óptico em VP; f. abertura em VE; g-h. análise L.O. em dois níveis de focalização. i-1. S. longipetiolata - i. corte óptico em VP; j. abertura em VE; k-1. análise L.O. em dois níveis de focalização. m-p. S. lucumoides - m. corte óptico em VP. N. Abertura em VE; o-p. análise L.O. em dois níveis de focalização. q-v. S. macrocarpa - q. corte óptico em VP; r. abertura em VE; s. vista equatorial em MEV (aumento 3.000x); t. vista polar em MEV (aumento 3.000x); u-v. análise L.O. em dois níveis de focalização. w-z. S. malmei - w. corte óptico em VP; x. abertura em VE; y-z. análise L.O. em dois níveis de focalização. a'-d'. S. morototoni a'. corte óptico em VP; b'. abertura em VE; c'-d'. análise L.O. em dois níveis de focalização. Escala $=5 \mu \mathrm{m}$. 


\begin{tabular}{|c|c|c|c|c|c|c|c|c|c|c|c|c|c|c|c|}
\hline \multirow[t]{2}{*}{ Especies } & \multicolumn{5}{|c|}{ Diametro polar (VE) } & \multicolumn{5}{|c|}{ Diametro equatorial (VE) } & \multicolumn{5}{|c|}{ Diametro equatorial (VP) } \\
\hline & $\begin{array}{l}\text { Faixa de } \\
\text { variação }\end{array}$ & $\bar{x} \pm s_{\bar{x}}$ & $s$ & $\mathrm{~V}(\%)$ & IC & $\begin{array}{l}\text { Faixa de } \\
\text { variação }\end{array}$ & $\bar{x} \pm s_{\bar{x}}$ & $s$ & $\mathrm{~V}(\%)$ & IC & $\begin{array}{l}\text { Faixa de } \\
\text { variação }\end{array}$ & $\bar{x} \pm s_{\bar{x}}$ & $s$ & $\mathrm{~V}(\%)$ & IC \\
\hline S. angustissima & $29,1-33,3$ & $30,9 \pm 0,19$ & 0,96 & 3,1 & $30,5-31,3$ & $30,5-34,6$ & $32,6 \pm 0,2$ & 0,99 & 3,03 & $32,2-33$ & $28,9-32,3$ & $30,4 \pm 0,24$ & 1,21 & 3,98 & $29,9-30,9$ \\
\hline S. calva & $27-29,9$ & $28,3 \pm 0,15$ & 0,76 & 2,69 & $27,9-28,6$ & $30,5-32,8$ & $31,5 \pm 0,11$ & 0,56 & 1,77 & $31,3-31,8$ & $27,5-29,9$ & $28,6 \pm 0,13$ & 0,63 & 2,2 & $28,4-28,9$ \\
\hline S. capixaba & $31,9-35,8$ & $33,7 \pm 0,19$ & 0,94 & 2,79 & $33,3-34,1$ & $32,1-35,3$ & $33,5 \pm 0,19$ & 0,93 & 2,78 & $33,1-33,9$ & $29,6-34,4$ & $32,1 \pm 0,26$ & 1,28 & 3,99 & $31,5-32,6$ \\
\hline S. cordata & $25-28,5$ & $26,8 \pm 0,15$ & 0,78 & 2,91 & $26,5-27,1$ & $27,1-30,4$ & $29 \pm 0,18$ & 0,93 & 3,21 & $28,6-29,4$ & $24,9-27,8$ & $26,4 \pm 0,14$ & 0,7 & 2,65 & $26,1-26,7$ \\
\hline S. fruticosa & $28,5-33,3$ & $31,3 \pm 0,26$ & 1,29 & 4,13 & $30,7-31,8$ & $30,3-34,4$ & $32,5 \pm 0,25$ & 1,27 & 3,91 & $32-33$ & $29,4-33,1$ & $31,3 \pm 0,22$ & 1,12 & 3,58 & $30,9-31,8$ \\
\hline S. gardneri & $26,5-33,3$ & $29,8 \pm 0,33$ & 1,66 & 5,58 & $29,1-30,5$ & $29-32,8$ & $31,2 \pm 0,19$ & 0,95 & 3,04 & $30,8-31,6$ & $26,9-31$ & $28,7 \pm 0,22$ & 1,09 & 3,8 & $28,2-29,1$ \\
\hline S. glaziovii & $27,4-31,4$ & $29,1 \pm 0,24$ & 1,18 & 4,05 & $28,6-29,6$ & $28,6-35,6$ & $31,3 \pm 0,41$ & 2,04 & 6,52 & $30,4-32,1$ & $26,6-31,3$ & $29,2 \pm 0,22$ & 1,1 & 3,76 & $28,8-29,7$ \\
\hline S. longipetiolata & $29,4-32,3$ & $30,6 \pm 0,18$ & 0,9 & 2,94 & $30,2-30,9$ & $31,4-35,6$ & $33,8 \pm 0,19$ & 0,98 & 2,9 & $33,4-34,2$ & $29,5-34,6$ & $32,2 \pm 0,28$ & 1,42 & 4,4 & $31,7-32,8$ \\
\hline S. lucumoides & $25,3-31,5$ & $27,6 \pm 0,12$ & 0,59 & 2,36 & $27,4-27,9$ & $26,9-31,3$ & $29 \pm 0,09$ & 0,45 & 0,8 & $28,8-29,2$ & $24,8-29,6$ & $27,5 \pm 0,1$ & 0,5 & 2 & $27,3-27,7$ \\
\hline S. macrocarpa & $28,4-32,3$ & $30,4 \pm 0,19$ & 0,95 & 3,13 & $30-30,7$ & $31-36$ & $33,2 \pm 0,31$ & 1,54 & 4,64 & $32,6-33,8$ & $29,1-33,6$ & $31,1 \pm 0,24$ & 1,2 & 3,85 & $30,6-31,6$ \\
\hline S. malmei & $21,5-24$ & $22,8 \pm 0,07$ & 0,35 & 1,4 & $22,7-22,9$ & $21,4-24,3$ & $23,1 \pm 0,08$ & 0,38 & 1,52 & $22,9-23,3$ & $19,6-24$ & $22 \pm 0,1$ & 0,48 & 1,92 & $21,8-22,2$ \\
\hline S. morototoni & $21-24,9$ & $22,9 \pm 0,09$ & 0,44 & 1,92 & $22,7-23,1$ & $22,8-25,5$ & $24,1 \pm 0,08$ & 0,14 & 0,58 & $23,9-24,3$ & $21,1-24,3$ & $22,7 \pm 0,08$ & 0,41 & 1,64 & $22,6-22,9$ \\
\hline S. selloi & $27,8-32,5$ & $29,6 \pm 0,22$ & 1,08 & 3,65 & $29,1-30$ & $30,6-35,1$ & $33,2 \pm 0,24$ & 1,20 & 3,62 & $32,7-33,7$ & $27,8-34,4$ & $31,4 \pm 0,26$ & 1,32 & 4,21 & $30,8-31,9$ \\
\hline S. spruceana & $31,6-37,5$ & $34,6 \pm 0,34$ & 1,71 & 4,94 & $33,9-35,3$ & $31,1-35,8$ & $34,2 \pm 0,23$ & 1,18 & 3,45 & $33,8-34,7$ & $31,6-36,5$ & $33,8 \pm 0,28$ & 1,42 & 4,2 & $33,3-34,4$ \\
\hline S. succinea & $25,4-33,8$ & $28,4 \pm 0,35$ & 1,77 & 6,23 & $27,7-29,1$ & $26,8-34,1$ & $30 \pm 0,33$ & 1,66 & 5,54 & $29,3-30,7$ & $25,9-30,9$ & $28,5 \pm 0,29$ & 1,44 & 5,05 & $27,9-29,1$ \\
\hline S. villosissima & $21,3-26,5$ & $23,9 \pm 0,1$ & 0,48 & 1,92 & $23,7-24,1$ & $22,8-26,5$ & $25 \pm 0,09$ & 0,44 & 1,76 & $24,8-25,2$ & $21,5-26,1$ & $23,5 \pm 0,1$ & 0,52 & 2,08 & $23,3-23,7$ \\
\hline S. vinosa & $26,8-31,4$ & $28,5 \pm 0,06$ & 1,22 & 4,28 & $28,1-28,6$ & $30-33,6$ & $32,1 \pm 0,2$ & 1 & 3,11 & $31,7-32,5$ & $28,3-31,6$ & $30 \pm 0,04$ & 0,96 & 3,2 & $29,9-30,1$ \\
\hline S. aff. varisiana & $30-35,4$ & $32,6 \pm 0,3$ & 1,49 & 4,56 & $32-33,3$ & $26,5-32$ & $29 \pm 0,27$ & 1,35 & 4,66 & $28,4-29,5$ & $25-32,1$ & $28,8 \pm 0,32$ & 1,6 & 5,55 & $28,2-29,5$ \\
\hline
\end{tabular}



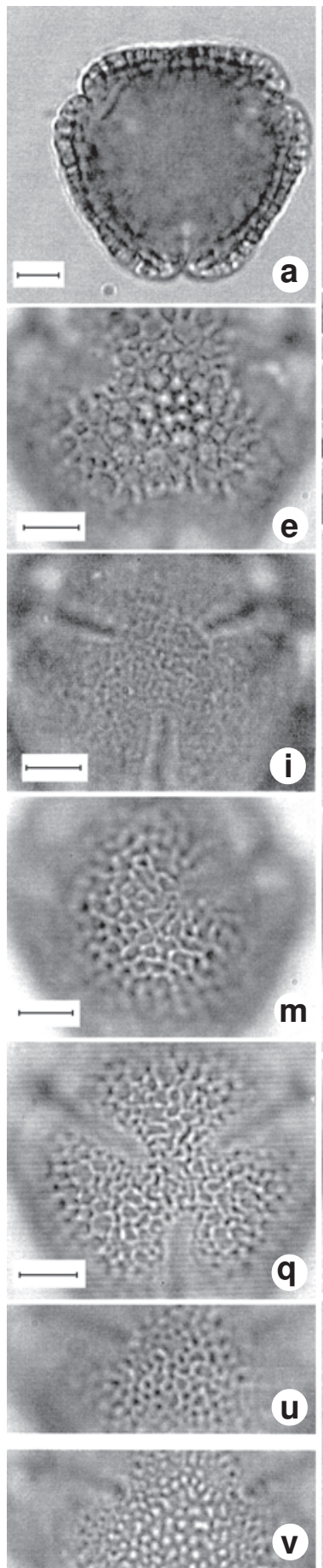
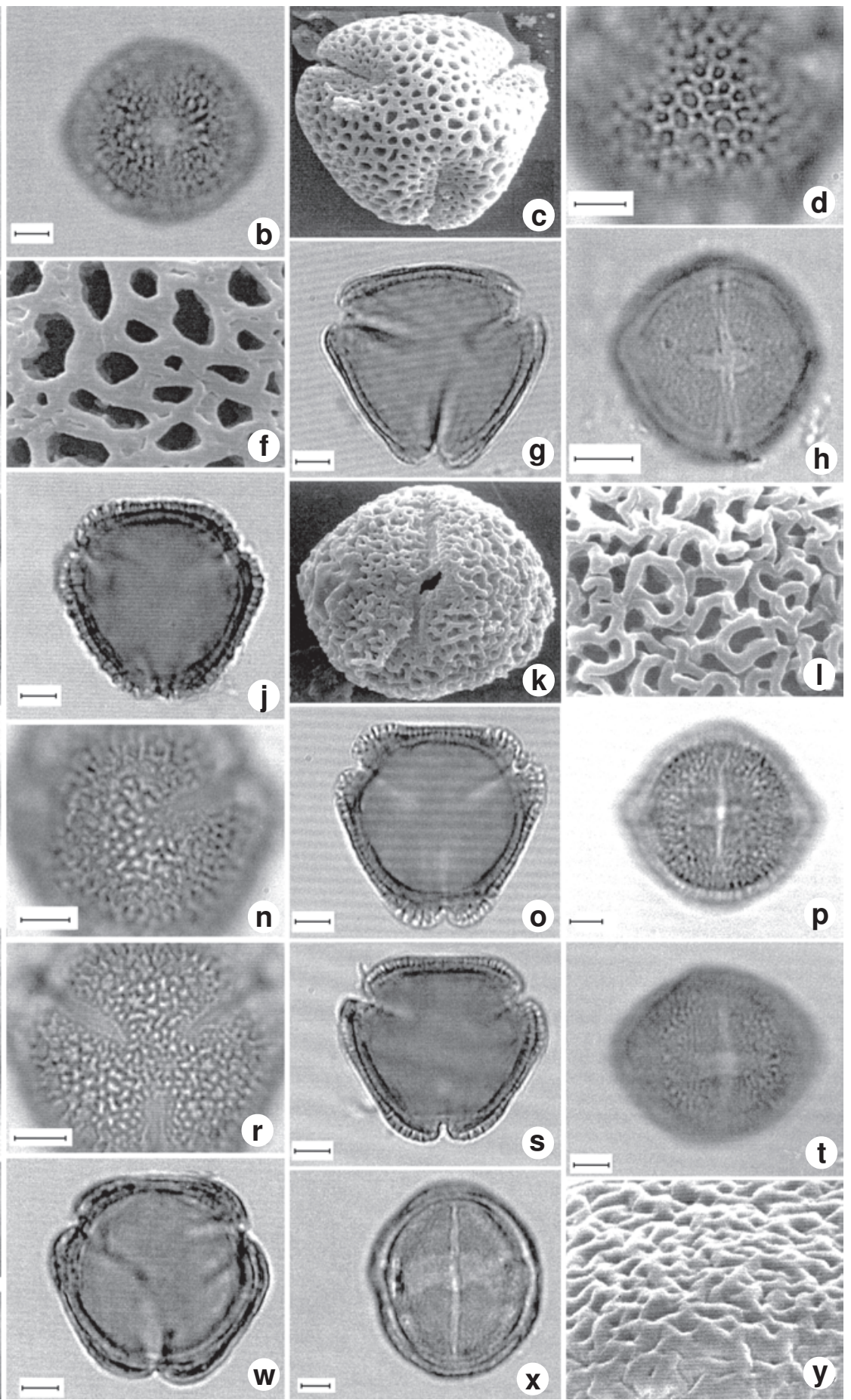

Figura 3 - Fotomicrografias e eletromicrografias dos grãos de pólen de Schefflera. a-f. S. selloi - a. corte óptico em VP; b. abertura em VE; c. vista polar em MEV (aumento 3.000x); d-e. análise L.O. em dois níveis de focalização; f. detalhe da ornamentação no apocolpo em MEV (aumento 10.000x). g-i. S. spruceana - g. corte óptico em VP; h. abertura em VE; i. detalhe do apocolpo em VP. j-n. S. succinea - j. corte óptico em VP; k. vista equatorial em MEV (aumento 3.000x); 1. detalhe da ornamentação no mesocolpo em MEV (aumento 10.000x); m-n. análise L.O. em dois níveis de focalização. o-r. S. villosissima - o. corte óptico em VP; p. abertura em VE; q-r. análise L.O. em dois níveis de focalização. s-v. S. vinosa - s. corte óptico em VP; t. abertura em VE; u-V. análise L.O. em dois níveis de focalização. w-y. S. aff. varisiana - w. corte óptico em VP; x. abertura em VE; y. detalhe da ornamentação no mesocolpo em MEV (aumento 10.000x). Escala = $5 \mu \mathrm{m}$. 
Tabela 3 - Média aritmética $(\mu \mathrm{m})$ das medidas do lado do apocolpo e das aberturas do grão de pólen de espécies de Schefflera $(\mathrm{n}=10)$.

\begin{tabular}{|c|c|c|c|c|c|c|}
\hline \multirow[t]{2}{*}{ Espécies } & \multirow{2}{*}{$\begin{array}{l}\text { Lado do } \\
\text { apocolpo }\end{array}$} & \multirow{2}{*}{$\begin{array}{c}\text { Índice de } \\
\text { área Polar (IAP) }\end{array}$} & \multicolumn{2}{|c|}{ Colpo } & \multicolumn{2}{|c|}{ Endoabertura } \\
\hline & & & Compr. & Larg. & Compr. & Larg. \\
\hline S. angustissima & 8,92 & 0,29 & 20,71 & 2,46 & - & - \\
\hline S. calva & 9,61 & 0,33 & - & - & - & - \\
\hline S. capixaba & 11,06 & 0,34 & 21,11 & - & - & - \\
\hline S. cordata & 8,92 & 0,34 & - & - & 5,00 & 8,98 \\
\hline S. fruticosa & 11,46 & 0,37 & 20,59 & 1,92 & $3,97 *$ & $10,78^{*}$ \\
\hline S. gardneri & 8,18 & 0,28 & $20,46^{*}$ & - & - & - \\
\hline S. glaziovii & 8,03 & 0,27 & $20,29 *$ & - & - & - \\
\hline S. longepetiolata & 10,88 & 0,34 & 20,78 & 1,88 & 2,67 & 11,03 \\
\hline S. lucumoides & 8,90 & - & $18,50^{*}$ & - & $2,00^{*}$ & - \\
\hline S. macrocarpa & 11,65 & 0,37 & $18,49 *$ & $2,31 *$ & $3,18^{*}$ & $9,93^{*}$ \\
\hline S. morototoni & 7,60 & - & - & - & - & - \\
\hline S. selloi & 9,51 & 0,30 & $18,06^{*}$ & - & - & - \\
\hline S. spruceana & 6,97 & 0,21 & 28,02 & 2,52 & 4,38 & 13,48 \\
\hline S. succinea & 7,97 & 0,28 & $21,14^{*}$ & $2,87 *$ & $4,87 *$ & $8,75^{*}$ \\
\hline S. villosissima & 7,12 & - & - & - & - & - \\
\hline S. vinosa & 9,25 & 0,31 & 19,31 & - & - & - \\
\hline S. aff. varisiana & 8,60 & 0,30 & 25,34 & 1,86 & 5,91 & 14,93 \\
\hline
\end{tabular}

$*_{\mathrm{n}} \leq 5$

Tabela 4 - Média aritmética da espessura $(\mu \mathrm{m})$ das camadas da exina dos grãos de pólen dos materiais padrão de espécies de Schefflera $(\mathrm{n}=10)$.

\begin{tabular}{lcccccc}
\hline Espécies & exina total & sexina & nexina total & nexina $\mathbf{1}$ & nexina 2 & teto \\
\hline S. angustissima & 2,68 & 1,31 & 1,30 & 0,38 & 0,92 & 0,48 \\
S. calva & 2,35 & 1,20 & 1,30 & 0,40 & 0,90 & 0,50 \\
S. capixaba & 3,51 & 1,99 & 1,55 & 0,43 & 1,12 & 0,59 \\
S. cordata & 2,93 & 1,33 & 2,14 & 0,47 & 1,67 & 0,73 \\
S. fruticosa & 3,67 & 1,52 & 2,23 & 0,77 & 1,46 & 0,82 \\
S. gardneri & 2,60 & 1,15 & 1,45 & 0,45 & 1,00 & 0,55 \\
S. glaziovii & 2,25 & 1,10 & 1,20 & 0,45 & 0,85 & 0,45 \\
S. longipetiolata & 3,38 & 1,43 & 2,29 & 0,84 & 1,45 & 0,91 \\
S. lucumoides & 2,73 & 1,27 & 2,00 & 0,47 & 1,53 & 0,60 \\
S. macrocarpa & 2,78 & 1,06 & 1,73 & 0,50 & 1,23 & 0,59 \\
S. malmei & 2,87 & 1,33 & 1,74 & 0,27 & 1,47 & 0,67 \\
S. morototoni & 3,73 & 2,00 & 2,20 & 0,40 & 1,80 & 0,93 \\
S. selloi & 3,25 & 1,45 & 1,85 & 0,65 & 1,20 & 0,75 \\
S. spruceana & 2,57 & 0,95 & 1,67 & 0,56 & 1,11 & 0,46 \\
S. succinea & 3,60 & 1,54 & 2,00 & 0,59 & 1,41 & 0,71 \\
S. villosissima & 3,27 & 1,47 & 2,07 & 0,40 & 1,67 & 0,73 \\
S. vinosa & 2,70 & 1,30 & 1,60 & 0,40 & 1,20 & 0,55 \\
S. aff. varisiana & 2,11 & 0,93 & 1,17 & 0,31 & 0,86 & 0,38 \\
\hline
\end{tabular}


Os grãos de pólen de Schefflera morototoni, anteriormente estudados por Pire (1989) e Sosa (1983), seguem o Tipo 1 aqui descrito. Seus grãos de pólen são oblatoesferoidais e possuem exina semitectada reticulada heterobrocada, com lumens de diferentes diâmetros (Fig. 2c'-d').

O outro padrão (Tipo 2), encontrado em apenas duas espécies (S. spruceana e $S$. aff. varisiana), caracteriza-se pelos grãos de pólen prolato-esferoidais e exina com ornamentação rugulado-reticulada (Fig. 3g-i, w-y). O compartilhamento de caracteres macromorfológicos, tais como a presença de nervuras intersecundárias evidentes, inflorescências com ramos secundários verticilados e flores com filetes mais longos que as anteras também sustenta seu agrupamento. Tanto S. spruceana quanto $S$. aff. varisiana pertencem ao grupo 'Crepinella' de Schefflera (Frodin 1995a), que abrange cerca de 40 espécies distribuídas essencialmente no norte da América do Sul, com centro de diversidade no Planalto das Guianas Venezuelano.

Desta maneira, dois dos grupos informais da classificação infragenérica de Schefflera nos neotrópicos (Frodin 1995a) são aqui sustentados com base na morfologia polínica: 'Didymopanax' e 'Crepinella', diferenciados, respectivamente, pelos grãos de pólen dos tipos 1 e 2 mencionados anteriormente.

Pode-se notar que as medidas dos diâmetros dos grãos de pólen do material de comparação (Tab. 6) muitas vezes não estão de acordo com a faixa de variação obtida para os materiaispadrão (Tab. 2). Tal discrepância sugere que o tamanho dos grãos de pólen constitui um caráter inconsistente para a palinotaxonomia das espécies aqui estudadas, assim como observado no estudo de Huang (1972). Em outros casos, porém, o tamanho dos grãos de pólen mostrou-se útil taxonomicamente na distinção de espécies pertencentes ao gênero (Melhem \& Bissa 1985; Tseng \& Shoup 1978).

Ao contrário das descrições apresentadas por Melhem \& Bissa (1985), segundo as quais foi possível distinguir $S$. angustissima
(=Didymopanax angustissimus Marchal) de S. calva (=Didymopanax micranthus Marchal) pelo tamanho dos grãos de pólen e detalhes da ornamentação na região do pólo, as análises aqui desenvolvidas com uma amostra mais significativa não nos permitiram chegar a conclusões semelhantes (Tabs. 2, 5, 6). Salgado-Labouriau (1973) ressaltou a existência de retículos e microrretículos misturados na região polar de $S$. vinosa [=Didymopanax vinosus (Cham. \& Schltdl.) Marchal], dados aqui corroborados, uma vez que todas as espécies reticuladas estudadas possuem lumens de diferentes proporções na região polar (Tab. 5).

Com relação à espessura das camadas da exina, os grãos de pólen das espécies estudadas (excetuando-se $S$. angustissima e $S$. capixaba) possuem a nexina mais espessa que a sexina, ao contrário da maioria das espécies descritas por Melhem \& Bissa (1985), Pire (1989), Salgado-Labouriau (1973) e Tseng $\&$ Shoup (1978).

Em um estudo com 48 espécies pertencentes ao gênero Schefflera, Tseng \& Shoup (1978) encontraram uma grande variedade de tipos polínicos, a partir dos quais reconheceram oito padrões morfológicos. Baseando-se na premissa de que as espécies 'primitivas' de Schefflera possuem flores polímeras (Eyde \& Tseng 1971), Tseng \& Shoup (1978) organizaram esses padrões polínicos num esquema de relações filogenéticas sugerindo que várias linhagens com grãos de pólen tectados e diversamente ornamentados derivaram de ancestrais com grãos de pólen tectado-psilados.

Por outro lado, a variabilidade polínica verificada por Tseng \& Shoup (1978) em Schefflera pode fornecer informações taxonômicas consistentes para a proposição de uma circunscrição mais precisa para o gênero, já que o mesmo constitui um grupo polifilético (Lowry et al. 2004; Plunkett et al. 2004, 2005; Wen et al. 2001). O reconhecimento de dois tipos polínicos distintos (Tipos 1 e 2) em espécies de Schefflera da Região Sudeste do Brasil 
Tabela 5-Amplitude de variação do número de lumens e quantidade de perfurações, microrretículos e retículos em $50 \mu \mathrm{m}^{2}$ de dez grãos de pólen de cada material padrão, com as respectivas porcentagens em parênteses.

\begin{tabular}{lcccc}
\hline Espécies & $\begin{array}{c}\text { número de } \\
\text { lumens }\end{array}$ & $\begin{array}{c}\text { perfurações } \\
(\mathbf{\%})\end{array}$ & $\begin{array}{c}\text { microrretículos } \\
(\mathbf{\%})\end{array}$ & $\begin{array}{c}\text { retículos } \\
(\mathbf{\%})\end{array}$ \\
\hline S. angustissima & $12-20$ & 0 & $55(35,71)$ & $99(64,29)$ \\
S. calva & $12-14$ & $2(1,53)$ & $37(28,24)$ & $92(70,23)$ \\
S. capixaba & $13-21$ & $1(0,61)$ & $56(34,15)$ & $107(65,24)$ \\
S. cordata & $20-24$ & 0 & $87(38,84)$ & $135(61,16)$ \\
S. fruticosa & $15-23$ & 0 & $75(38,86)$ & $118(61,14)$ \\
S. gardneri & $24-33$ & $7(2,46)$ & $219(77,11)$ & $58(20,43)$ \\
S. glaziovii & $21-26$ & $3(1,29)$ & $163(69,96)$ & $67(28,76)$ \\
S. longipetiolata & $21-25$ & $6(2,65)$ & $129(57,08)$ & $91(40,27)$ \\
S. lucumoides & $19-31$ & 0 & $225(81,82)$ & $50(18,18)$ \\
S. macrocarpa & $15-24$ & 0 & $119(60,71)$ & $77(39,29)$ \\
S. malmei & $15-23$ & 0 & $78(42,86)$ & $104(57,14)$ \\
S. morototoni & $14-20$ & $4(2,41)$ & $68(40,96)$ & $94(56,63)$ \\
S. selloi & $10-17$ & $1(0,69)$ & $40(27,78)$ & $101(71,53)$ \\
S. succinea & $15-27$ & $1(0,5)$ & $107(53,23)$ & $93(46,27)$ \\
S. villosissima & $19-28$ & $1(0,43)$ & $147(63,36)$ & $84(36,21)$ \\
S. vinosa & $22-30$ & $2(0,77)$ & $230(88,12)$ & $29(11,11)$ \\
\hline
\end{tabular}

Tabela 6 - Média aritmética $(\mu \mathrm{m})$ das medidas dos grãos de pólen dos materiais de comparação de espécies de Schefflera $(\mathrm{n}=10)$.

\begin{tabular}{lcccc}
\hline Espécies & \multicolumn{2}{c}{$\begin{array}{c}\text { Vista equatorial } \\
\text { Diâmetro } \\
\text { polar }(\boldsymbol{\mu m})\end{array}$} & $\begin{array}{c}\text { Vista polar } \\
\text { equatorial }(\boldsymbol{\mu m})\end{array}$ & $\begin{array}{c}\text { P/E } \\
\text { Diâmetro } \\
\text { equatorial }(\boldsymbol{\mu m})\end{array}$ \\
\hline S. angustissima & 31,6 & 33,5 & 31,1 & 0,94 \\
Costa 742 & 23,1 & 24,5 & 23,3 & 0,94 \\
Handro 96 & 33,0 & 34,9 & 30,8 & 0,95 \\
Ivanauskas 559 & 27,0 & 27,1 & 26,3 & 1,00 \\
Martins 601 & & & & \\
S. calva & 29,4 & 29,6 & 28,1 & 0,99 \\
Pirani CFSC 11027 & & & & \\
S. fruticosa & $26,1 *$ & $27,0 *$ & 26,1 & 0,97 \\
Roque 104 & & & & \\
S. gardneri & 28,6 & 28,3 & 28,3 & 1,01 \\
Furlan CFCR 3224 & 29,1 & 29,9 & 28,5 & 0,97 \\
Pirani 4383 & & & & \\
S. glaziovii & 26,1 & 28,5 & 25,7 & 0,91 \\
Fiaschi 330 & 27,6 & 29,5 & 28,0 & 0,93 \\
Fiaschi 343 & & & & \\
S. longipetiolata & 29,4 & 30,0 & 29,9 & 0,98 \\
Andrade 348 & & & & \\
\hline
\end{tabular}




\begin{tabular}{|c|c|c|c|c|}
\hline \multirow[t]{2}{*}{ Espécies } & \multicolumn{2}{|c|}{ Vista equatorial } & \multirow{2}{*}{$\begin{array}{c}\text { Vista polar } \\
\text { Diâmetro } \\
\text { equatorial }(\mu \mathrm{m})\end{array}$} & \multirow[t]{2}{*}{$\mathbf{P} / \mathbf{E}$} \\
\hline & $\begin{array}{l}\text { Diâmetro } \\
\text { polar }(\mu \mathrm{m})\end{array}$ & $\begin{array}{c}\text { Diâmetro } \\
\text { equatorial }(\mu \mathrm{m})\end{array}$ & & \\
\hline Andrade 511 & 31,6 & 32,3 & 31,0 & 0,98 \\
\hline Farney 1444 & $28,7^{*}$ & $30,5^{*}$ & 28,4 & 0,94 \\
\hline Kuhlmann s.n. (RB 21344) & $29,4^{*}$ & $30,2^{*}$ & 28,9 & 0,97 \\
\hline \multicolumn{5}{|l|}{ S. lucumoides } \\
\hline Pinto s.n. (BHCB 11726) & $28,0^{*}$ & $30,8^{*}$ & 28,9 & 0,91 \\
\hline Teixeira s.n. (BHCB 26039) & 30,4 & 31,5 & 29,6 & 0,96 \\
\hline \multicolumn{5}{|l|}{ S. macrocarpa } \\
\hline Fiaschi 140 & 31,0 & 31,4 & 29,4 & 0,99 \\
\hline Giulietti CFSC 7909 & 27,3 & 31,2 & 28,9 & 0,87 \\
\hline Guedes PCD 5421 & 25,1 & 26,3 & 25,6 & 0,96 \\
\hline Proença 2162 & 29,1 & 31,7 & 29,7 & 0,92 \\
\hline \multicolumn{5}{|l|}{ S. selloi } \\
\hline Belém 3154 & 25,1 & 29,0 & 27,8 & 0,87 \\
\hline Mattos-Silva 1807 & 28,3 & 28,6 & 27,9 & 0,99 \\
\hline Peixoto 379 & 28,8 & 30,8 & 29,0 & 0,93 \\
\hline \multicolumn{5}{|l|}{ S. succinea } \\
\hline Martinelli 13395 & 31,0 & 32,0 & 30,6 & 0,97 \\
\hline \multicolumn{5}{|l|}{ S. vinosa } \\
\hline Conceição PCD 2218 & 29,1 & 30,5 & - & 0,95 \\
\hline Fiaschi 350 & 26,9 & 28,7 & 28,3 & 0,94 \\
\hline Forzza 1148 & 26,1 & 28,2 & 26,2 & 0,92 \\
\hline Harley s.n. (SPF 33280) & 24,0 & 26,1 & 25,1 & 0,96 \\
\hline Pirani CFCR 7932 & 27,1 & 29,2 & 27,0 & 0,93 \\
\hline \multicolumn{5}{|l|}{ S. aff. varisiana } \\
\hline Harley H 52026 & 28,0 & 26,9 & 25,3 & 1,04 \\
\hline Laessoe H 53300 & 32,9 & 29,5 & - & 1,11 \\
\hline Sano H 52190 & 28,4 & 24,6 & 23,9 & 1,15 \\
\hline Stannard H 51151 & 32,3 & 29.9 & $29,9 *$ & 1,08 \\
\hline
\end{tabular}

$* \mathrm{n} \leq 5$

também sugere que estudos mais abrangentes possam fornecer dados taxonômicos valiosos para uma classificação infragenérica dos representantes neotropicais do gênero.

Das poucas espécies neotropicais de Schefflera cujos grãos de pólen foram analisados por Tseng \& Shoup (1978), a que mais se assemelha às espécies aqui analisadas é $S$. decaphylla (Seem.) Harms (=S. paraensis Hub. ex Ducke). Segundo Shoup \& Tseng (1977), secções dos grãos de pólen de $S$. decaphylla evidenciam um tipo incomum de sexina com columelas fundidas lateralmente formando unidades cilíndricas, quando analisadas em MEV. Embora não tenha sido possível constatar a ocorrência dessa ornamentação nas espécies aqui estudadas, foi possível verificar que a morfologia polínica de $S$. decaphylla é muito semelhante à das espécies do grupo 'Didymopanax' aqui descritas sob MEV (Tipo 1). Assim, provavelmente todas as espécies aqui analisadas, cujos grãos de pólen são reticulados heterobrocados, compartilham com S. decaphylla o mesmo padrão de 
ornamentação. Isso corroboraria, apesar de possuir flores com o ovário 5-locular, com o posicionamento desta espécie no grupo 'Didymopanax', diferentemente da maioria das demais espécies, cujas flores têm o ovário geralmente 2-locular (Frodin 1995a).

\section{Agradecimentos}

Os autores agradecem à FAPESP, ao CNPq (bolsa GDE \# 200682/2006-7) e ao programa de doutoramento em 'Integrative Life Sciences' da Virginia Commonwealth University (VCU) pelo apoio financeiro, e à Fundação Botânica Margaret Mee e ao IAPT (International Association for Plant Taxonomy) pelo apoio financeiro complementar. Aos curadores dos herbários BHCB, ESA, MBM, RB, SP, SPF, UB e UEC, que permitiram a remoção de material para o desenvolvimento desse estudo, e ao Instituto de Biociências da USP, pela preparação do material e obtenção das microfotografias em MEV.

\section{REFERÊNCIAS BIBLIOGRÁFICAS}

Barth, O. M. \& Melhem, T. S. 1988. Glossário ilustrado de palinologia. Editora da Unicamp, Campinas, 75p.

Erdtman, G. 1960. The acetolysis method. A revised description. Svensk Botanisk Tidskrift 54: 561-564.

Eyde, R. H. \& Tseng, C. C. 1971. What is the primitive floral structure of Araliaceae? Journal of the Arnold Arboretum, Harvard University 52: 205-239.

Fiaschi, P. 2004. Schefflera aurata, a new species of Araliaceae from Southern Bahia. Brittonia 56: 357-360.

; Frodin, D. G. \& Plunkett, G. M. 2008.

Four new species of the Didymopanax group of Schefflera (Araliaceae) from the Brazilian Amazon. Brittonia 60: 274-286.

\& Pirani, J. R. 2005a. Four new species of Schefflera (Araliaceae) from Espírito Santo State, Brazil. Kew Bulletin 60: 77-85.

\& Pirani, J. R. 2005b. Three new species of Schefflera J.R. Forst. \& G.
Forst. (Araliaceae) from Espinhaço Range, Minas Gerais, Brazil. Novon 15: 117-122.

\& Pirani, J. R. 2005c. Flora da Serra de Cipó, Minas Gerais: Araliaceae. Boletim de Botânica da Universidade de São Paulo 23: 267-275.

\& Pirani, J. R. 2007. Estudo taxonômico do gênero Schefflera J.R. Forst \& G. Forst. (Araliaceae) na Região Sudeste do Brasil. Boletim de Botânica da Universidade de São Paulo 25: 95-142.

Frodin, D. G. 1993. Studies in Schefflera (Araliaceae), VI. New species and subordinate taxa in the Venezuelan Guayana and immediately adjacent areas. Novon 3:367-403.

1995a. Neotropical Montane Araliaceae: an Overview. In: Churchill, S. P., Balslev, H., Forero, E. \& Luteyn, J. L. Biodiversity and conservation of neotropical montane forests. New York Botanical Garden, New York. Pp. 421-430. 1995b. Araliaceae. In: Stannard, B. L. Flora of the Pico das Almas - Chapada Diamantina, Bahia, Brazil. Royal Botanic Gardens, Kew. Pp. 138-140.

1997. Araliaceae. In: Steyermark, J. A.; Berry, P. E. \& Holst, B. K. Flora of the Venezuelan Guayana. Vol. 3. Missouri Botanical Garden, St. Louis. Pp. 1-131.

2004. Araliaceae. In: Smith, N., Mori, S. A., Henderson, A., Stevenson, D. W. $\&$ Heald, S. V. Flowering plants of the neotropics. Princeton University Press, Princeton. Pp. 28-31.

\& Govaerts, R. 2003. World checklist and bibliography of Araliaceae. The Royal Botanic Gardens, Kew, 444p.

Holmgren, P. K., Holmgren, N. H. \& Barnett, L. C. 1990. Index Herbariorum, Part I: The Herbaria of the World. New York Botanical Garden, New York, 693p.

Huang, T. C. 1972. Pollen Flora of Taiwan. National Taiwan University, Botany Dep. Press, Taipeh, 297p.

Jung, S. L. 1981. Flora fanerogâmica da Reserva do Parque Estadual das Fontes 
do Ipiranga (São Paulo, Brasil): Araliaceae. Hoehnea 9: 112-114.

Jung-Mendaçolli, S. L. \& Cabral, L. P. 2000. Araliaceae. In: Melo, M. M. R. F.; Barros, F.; Wanderley, M. G. L.; Kirizawa, M.; Jung-Mendaçolli, S. L. \& Chiea, S. A. C. Flora fanerogâmica da Ilha do Cardoso. Vol. 7. Instituto de Botânica, São Paulo. Pp. 11-16.

Lowry II, P. P.; Plunkett, G. M. \& Wen, J. 2004. Generic relationships in Araliaceae: looking into the crystal ball. South African Journal of Botany 70: 382-392.

Maguire, B.; Steyermark, J. A. \& Frodin, D. G. 1984. Araliaceae, p. 46-82. In: Maguire, B.; Cowan, R. S.; Wurdack, J. J. \& collaborators. The Botany of the Guayana Highland - Part XII. Memoirs of the New York Botanical Garden 38: 1-84.

Melhem, T. S. \& Bissa, W. M. 1985. Flora polínica da Reserva do Parque Estadual das Fontes do Ipiranga: Família 130Araliaceae. Hoehnea 12: 16-19.

; Cruz-Barros, M. A. V.; Corrêa, A. M. S.; Makino-Watanabe, H.; SilvestreCapelato, M. S. F. \& Esteves, V. L. G. 2003. Variabilidade polínica em plantas de Campos de Jordão (São Paulo, Brasil). Boletim do Instituto de Botânica, São Paulo 16: 1-104.

Pire, S. M. 1989. Morfologia polinica de las Araliaceae de Argentina. Bonplandia 6: 133-150.

Plunkett, G. M.; Wen, J. \& Lowry II, P. P. 2004. Infrafamilial classifications and characters in Araliaceae: Insights from the phylogenetic analysis of nuclear (ITS) and plastid (trnL-trnF) sequence data. Plant Systematics and Evolution 245: 1-39.

; Lowry II, P. P.; Frodin, D. G. \& Wen, J. 2005. Phylogeny and geography of Schefflera: pervasive polyphyly in the largest genus of Araliaceae. Annals of the Missouri Botanical Garden 92: 202-224.

Punt, W., Hoen, P. P., Blackmore, S., Nilsson, S. \& Le Thomas, A. 2007. Glossary of pollen and spore terminology. Review of Palaeobotany and Palynology 143: 1-81.

Salgado-Labouriau, M. L. 1973. Contribuição à palinologia dos cerrados. Academia Brasileira de Ciências, Rio de Janeiro, 273p. -; Vanzolini, P. E. \& Melhem, T. S. 1965. Variation of polar axes and equatorial diameters in pollen grains of two species of Cassia. Grana Palynologica 6: 166-176.

Shoup, J. R. \& Tseng, C. C. 1977. A Palynological study of Schefflera paraensis Huber ex Ducke (Araliaceae). Grana 16: 81-84.

Sosa, V. 1983. Características palinológicas de las araliáceas de México. Boletin de la Sociedad Botánica de México 45: 117-132.

Tseng, C. C. \& Shoup, J. R. 1978. Pollen morphology of Schefflera (Araliaceae). American Journal of Botany 65: 384-394.

Vieira, S. 1981. Introdução à bioestatística. Editora Campus, Rio de Janeiro, 294p.

Wen, J., Plunkett, G. M., Mitchell, A. D. \& Wagstaff, S. J. 2001. The Evolution of Araliaceae: A Phylogenetic Analysis Based on ITS Sequences of Nuclear Ribosomal DNA. Systematic Botany 26: 144-167. 